\title{
Ganglionic eminence within the early developing brain visualized by 3D transvaginal ultrasound
}

\author{
Dan Boitor-Borza, Tunde Kovacs, Florin Stamatian
}

IMOGEN Institute of Research, $1{ }^{\text {st }}$ Obstetrics and Gynecology Department, "Iuliu Hatieganu" University of Medicine and Pharmacy, Cluj-Napoca, Romania

\begin{abstract}
Aim: Early diagnosis of cerebral congenital anomalies requires a profound knowledge of the anatomy of the developing human brain. The ganglionic eminences (GE) are crucial structures of the brain, giving rise mostly to the basal nuclei. The aim of this explorative study is to assess the GE within the embryonic and early fetal brain by using 3D transvaginal US. Material and methods: From March 2015 to May 2015, a total of 18 singleton non-malformed embryos and fetuses at 9-13 weeks of gestation were assesed in vivo by transvaginal ultrasound using a Voluson E10, BT 15 scanner (GE Healthcare, Zipf, Austria). The 3D sonography was performed routinely as the subjects were scanned. Inter-observer agreement (concordance) was calculated using the Cohen's kappa statistics. Results: At 9 gestational weeks, no GE was identified. At 10 gestational weeks the GE were identified as mere thickenings in the lateral wall of the cerebral hemispheres, well depicted by 3D transvaginal ultrasound using the HDlive rendering mode and the OmniView ${ }^{\circledR}$ software. Starting with 11 gestational weeks the GE are evident. The results of inter-observer agreement for GE identification were as follows: observed agreement $P_{0}=0.94$, expected agreement $P \mathrm{e}=0.76$, kappa coefficient $=0.83$, which means a very good agreement between the observers. Conclusions: The GE can be clearly visualized by 3D transvaginal sonography, and especially by HDlive rendering mode. This method has become the "golden standard" for in vivo morphological studies of the embryonic and early fetal brain.
\end{abstract}

Keywords: ganglionic eminence, human embryo, transvaginal ultrasound

\section{Introduction}

The brain is one of the first organs to form and the last to accomplish its development. The embryology of the central nervous system is involved in human pathology, yet studying morphogenesis is also of interest in the fields of education and developmental sciences. Nowadays, many research teams are focused on diagnosing the congenital anomalies by ultrasound (US) in the first trimester of pregnancy. This requires, however, a profound knowledge of the normal anatomy of the developing human brain [1].

In our era, when most aspects of daily life tend to become computerized, the need to review the methods

Received 13.05.2015 Accepted 02.06.2015

Med Ultrason

2015, Vol. 17, No 3, 289-294

Corresponding author: Dan Boitor-Borza

1st Obstetrics and Gynecology Department,

3-5 Clinicilor Street,

400006 Cluj-Napoca, Romania

E-mail: dan.boitor@umfcluj.ro employed in classical embryological research is becoming more apparent. Considering recent improvements in technology, the human embryonic brain can be currently studied in many different aspects.

The modern US techniques and computer technology enable the 3D virtual reconstruction of the embryonic brain. Using a transvaginal 3D transducer is particularly important in the first trimester of pregnancy due to the impossibility to obtain all planes using only $2 \mathrm{D}$ transducer [2]. 3D/4D HDlive, as a surface render mode, uses an adjustable light source, giving the operator the opportunity to create lighting and shadowing effects and thereby increasing depth perception [3].

The ganglionic eminences (GE) present as thickenings of the lateral wall and floor of the third and lateral ventricles at 5 weeks post-fertilization (Carnegie stages 14 and 15) [4]. These are crucial structures in the embryonic and fetal brain, giving rise to the basal nuclei and the GABAergic cortical interneurons which will tangentially migrate towards the cortical plate [5]. The GE 

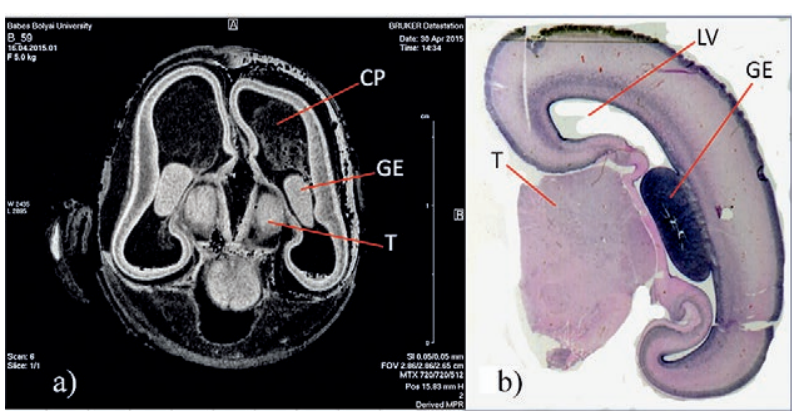

Fig 1. a) Human fetus 12 GW imaged by micro-MRI, axial section; b) microscopic photograph $(4 \mathrm{x})$ of a histological transverse section through the right cerebral hemisphere of a human fetus 14 post-conceptional weeks, hematoxylin-eosin staining. GE: ganglionic eminence, $\mathrm{CP}$ : choroid plexus within the lateral ventricle, T: thalamus, $\mathrm{LV}$ : anterior horn of the lateral ventricle.

are transient nervous structures which will disappear up to the $35^{\text {th }}$ week of gestation. Müller and O'Rahilly [6] demonstrated that, in fact, the GE are composed of two distinct structures of different origins, medial ventricular eminence of diencephalic origin and lateral ventricular eminence of telencephalic origin. The GE are characterized by large size and the exceptional persistence of their subventricular layer $[4,6]$. The GE can be clearly depicted by micro-MRI at 9 weeks of gestation [7] (fig 1).

During the past decades, the human embryonic brain in particular has been intensively studied $[1,8,9]$. To the best of our knowledge, imaging GE by US in the first trimester of pregnancy has not been reported in literature to date.

The aim of this explorative study is to assess the GE within the embryonic and early fetal brain by using 3D transvaginal US.

\section{Material and methods}

\section{Human embryos and fetuses}

From March 2015 to May 2015, a total of 18 singleton non-malformed embryos and fetuses at 9-13 weeks of gestation were studied. The gestational age was calculated by the crown-rump length (CRL) of the subjects and was expressed as gestational weeks (GW) - completed weeks from the last menstrual period.

The study protocol was approved by the local Ethics Committee. Written informed consent was obtained from each patient before the procedure, according to the World Medical Association Declaration of Helsinki, revised in 2000, Edinburgh.

\section{Acquisition of images}

The embryos and fetuses were assesed in vivo by transvaginal US using a Voluson E10, BT 15 ultrasound scanner (GE Healthcare, Zipf, Austria). The 3D US including the 3D HDlive rendering mode was performed routinely as the subjects were scanned. Using a mechanical high-frequency transvaginal transducer $(6-12 \mathrm{MHz} /$ 256-element 3D/4D), the aquisition of the volume took a few seconds at an angle of $70^{\circ}-85^{\circ}$. All examinations were performed by the same investigator.

\section{Image post-processing}

All volume data were reviewed retrospectively, and the optimal volumes were selected for further analysis. The criteria of inclusion of the optimal volumes were: absence of movement artifacts and accurate visualization of the structures around the GE, i.e. choroid plexus within the lateral ventricles and thalamus.

Two authors independently examined the optimal volumes using the 3D HDlive render mode and the Omni view ${ }^{\circledR}$ software (GE Healthcare, Zipf, Austria), which was employed for digitally slicing the selected volumes. Sections corresponding to the three orthogonal planes and "anyplane" sections were made in order to better visualize the GE. The Silhouette ${ }^{\circledR}$ mode was used for underlying the walls of the cerebral cavities.

GE were identified considering their anatomical characteristics described in classical papers of embryology $[4,6]$ and their micro-MRI appearance [7].

\section{Statistical analysis}

Data were expressed as minimum and maximum values obtained. Inter-observer agreement (concordance) was calculated using the Cohen's kappa statistics. Data evaluation and statistical analysis were performed with Office Excel SPSS program 19.0 version.

\section{Results}

The characteristics of the analyzed embryos and fetuses are detailed in table I.

The clear visualization of the GE with the OmniView ${ }^{\circledR}$ software and the HDlive rendering mode was possible in 15 out of 18 optimal volumes.

The results of inter-observer agreement for GE identification were as follows: observed agreement $P_{\mathrm{O}}=0.94$, expected agreement $P \mathrm{e}=0.76$, kappa coefficient $=0.83$, which means a very good agreement between the observers.

At $9 \mathrm{GW}$ no GE was identified.

Table I. Characteristics of the subjects

\begin{tabular}{llllll}
\hline Week of gestation & 9 & 10 & 11 & 12 & 13 \\
Number of subjects & 3 & 4 & 3 & 3 & 5 \\
CRL (mm) & $23-29$ & $31-38$ & $42-50$ & $55-64$ & $67-79$ \\
\hline
\end{tabular}

Results are expressed as minimum - maximum of crown-rump length (CRL) of the subjects. 
At $10 \mathrm{GW}$ the GE were identified as mere thickenings in the lateral wall of the cerebral hemispheres, well depicted by 3D transvaginal ultrasound using the HDlive rendering mode (fig 2) and the OmniView ${ }^{\circledR}$ software (fig 3). Starting with $11 \mathrm{GW}$ the GE are evident and can be identified ventrally to the choroid plexuses, bulging into the lateral ventricles, naturally visualized by the HDlive rendering mode (fig 4 a,b, fig 5 a,b, fig. 6). The GE are located rostrally to the thalamus, as seen on $2 \mathrm{D}$ images processed with the OmniView ${ }^{\circledR}$ software (fig 3; fig 4 c,d, fig 5 c,d, fig 7).

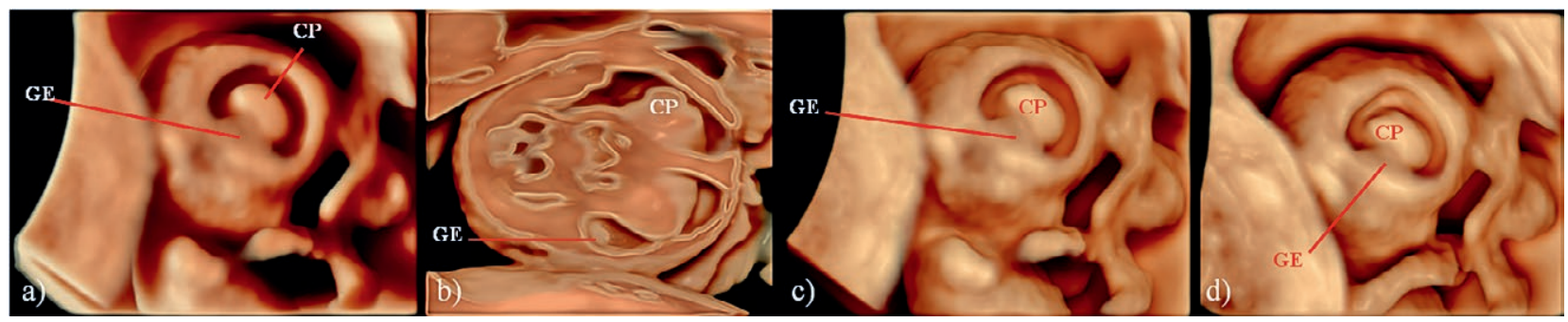

Fig 2. Human fetus $10 \mathrm{GW}$ imaged by 3D HDlive rendering mode. Varying different technical parameters (contrast, brightness, threshold) allows to obtain anatomical realistic images $(a, c, d)$. Silhouette ${ }^{\circledR}$ mode was employed in order to clearly delimitate the walls of the lateral ventricles (b). GE: ganglionic eminence, CP: choroid plexuses within the lateral ventricle.

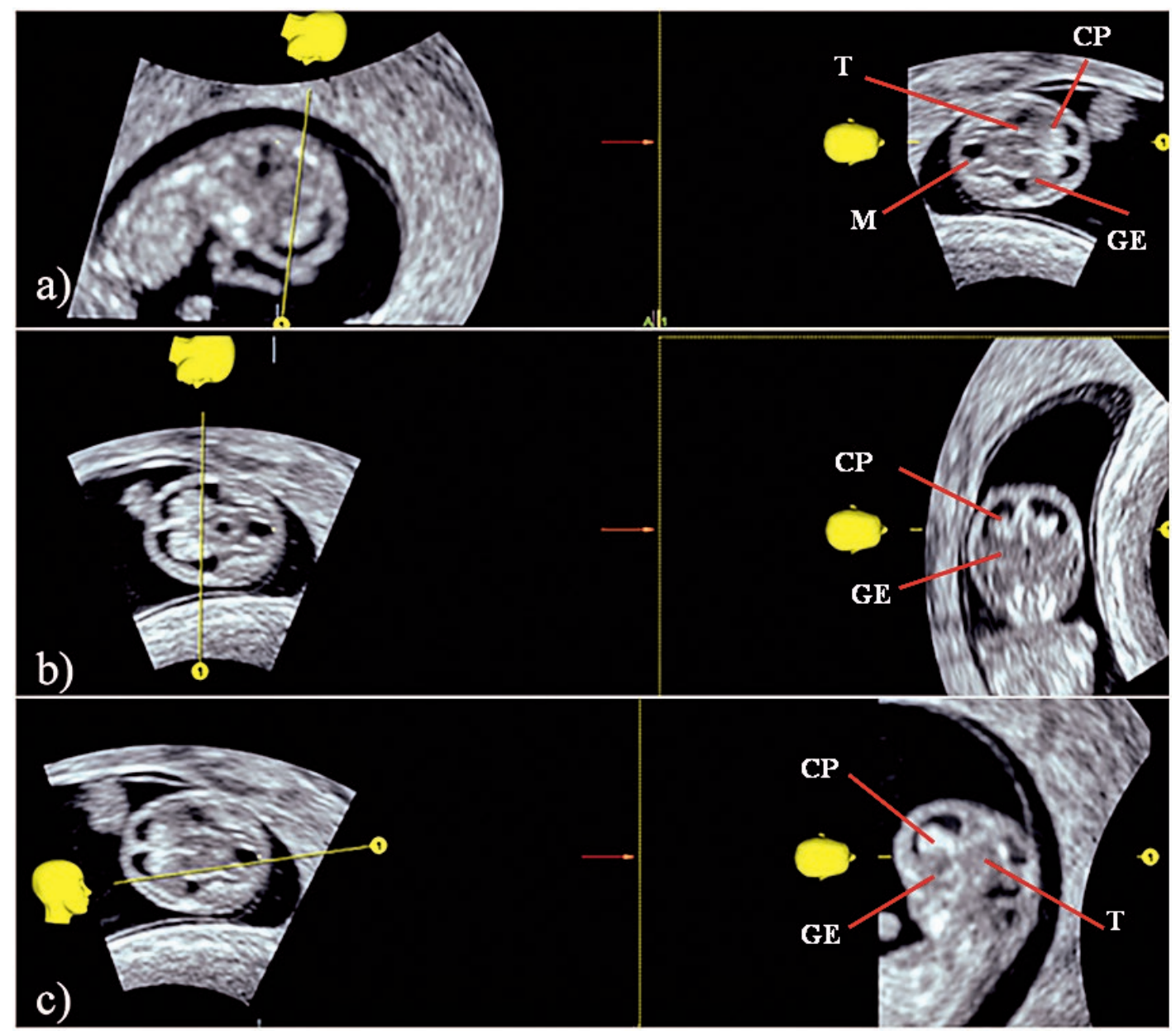

Fig 3. Human fetus $10 \mathrm{GW}$ imaged by $3 \mathrm{D}$ transvaginal ultrasound. OmniView ${ }^{\circledR}$ software was employed for slicing the acquired volume. Axial (a), coronal (b) and "anyplane" (c) sections are shown. GE: ganglionic eminences, $\mathrm{CP}$ : choroid plexuses within the lateral ventricle, T: thalamus, M: mesencephalon. 


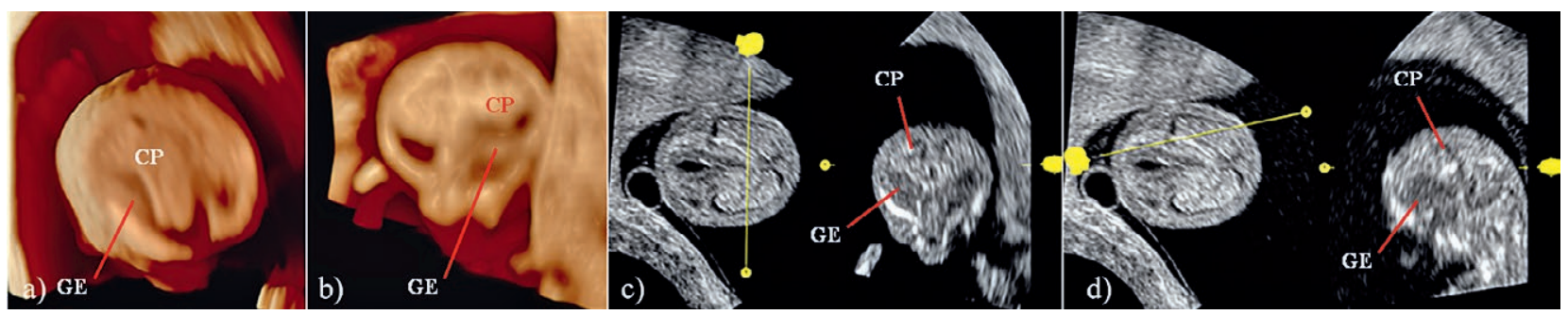

Fig 4. Human fetus $11 \mathrm{GW}$ imaged by $3 \mathrm{D}$ transvaginal ultrasound. HDlive rendering mode (a,b) and OmniView ${ }^{\circledR}(\mathrm{c}, \mathrm{d})$ images are shown. Right lateral view (a), left lateral view (b), coronal section (c) and "anyplane" section (d) are presented. GE: ganglionic eminences, CP: choroid plexuses within the lateral ventricles.
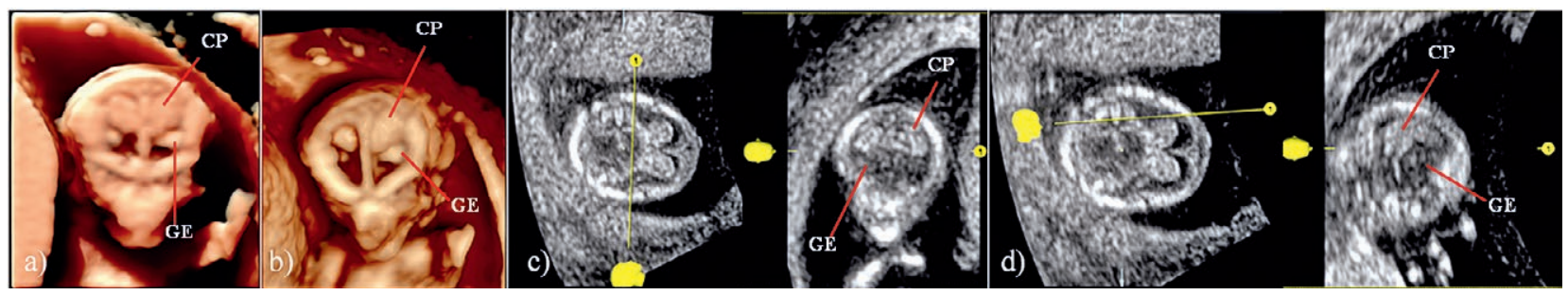

Fig 5. Human fetus $12 \mathrm{GW}$ imaged by $3 \mathrm{D}$ transvaginal ultrasound. HDlive rendering mode (a,b) and OmniView ${ }^{\circledR}(\mathrm{c}, \mathrm{d})$ images are shown. Coronal view (a), left lateral view (b), coronal slice (c) and "anyplane" slice (d) are presented. GE: ganglionic eminences, $\mathrm{CP}$ : choroid plexuses within the lateral ventricles.
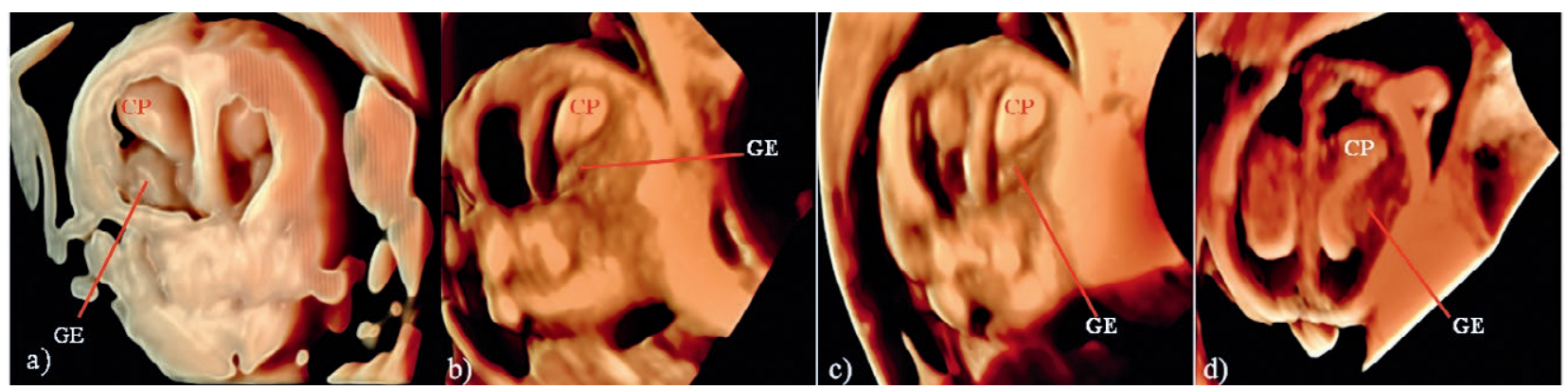

Fig 6. Human fetus $13 \mathrm{GW}$ imaged by 3D HDlive rendering mode. Coronal (a), left lateral (b,c) and axial (d) views are shown. GE: ganglionic eminence, CP: choroid plexus, LV: anterior horn of the lateral ventricle.
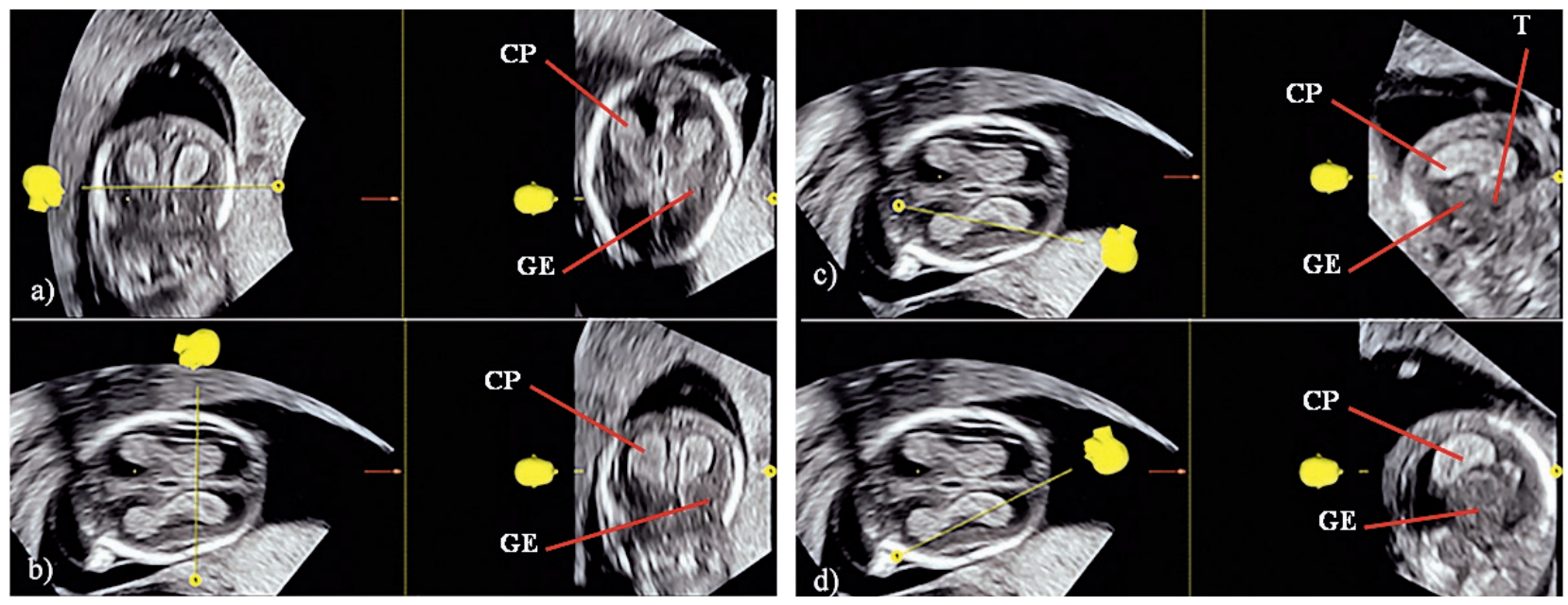

Fig 7. Human fetus $13 \mathrm{GW}$ imaged by $3 \mathrm{D}$ transvaginal ultrasound. OmniView ${ }^{\circledR}$ software was employed for slicing the acquired volume. Axial (a), coronal (b) and "anyplane" (c,d) sections are shown. GE: ganglionic eminence, CP: choroid plexus, 3rdV: third ventricle, T: thalamus. 


\section{Discussions}

Our explorative study demonstrates that the GE can be assessed by 3D transvaginal US from $10 \mathrm{GW}$ onwards, either on 2D sections or using the HDlive rendering mode. This method provides accurate images of small-sized structures of the developing brain, such as the GE.

3D/4D HDlive rendering mode provides us with a natural and anatomically realistic appearance of the embryo [10]. With this method, the images of the embryo seem to be more readily discernible than those obtained by conventional 3D sonography [11].

In literature we have found papers on fetal, placental and umbilical cord ultrasound using the HDlive rendering mode $[3,12,13]$, but none of these reported the imaging of the GE within the embryonic and early fetal brains.

3D HDlive rendering mode shows some advantages for the assessment of embryos and fetuses in the first trimester, compared to 2D US. Those advantages are: better visualization of fetal anatomy in multiplanar mode, shorter exposure time of the embryo to the ultrasound system $[11,14,15]$, the possibility of storage of the volumes, and its later processing and analysis without the presence of the patient [15].

We could not depict the GE clearly in embryos at 9 GW. This was due to the small size of those structures at the mentioned gestational age. Among the limitations of 3D US, the artifacts caused by embryonic or maternal movement, which reduce the quality of the image, should be mentioned [16]. The quantity of the cerebrospinal fluid inside the lateral ventricles could also influence the quality of rendered images.

Fetal neuroimaging with advanced 3D US technology is an easy, non-invasive and reproducible method which enables easy off-line analyses of the raw volume data sets [17]. This method has moved the prenatal diagnosis of fetal abnormalities from the second to the first trimester of pregnancy [18].

The good inter-observer agreement is important because the GE are small-sized structures which need to be precisely identified within the embryonic brain.

Identification of GE in early life is useful as they are crucial nervous structures which could be involved in teratology. In fact, at $6 \mathrm{GW}$ the neural anomalies are 40 times more frequent than at birth, which means that most of the affected conceptuses are lost until birth, and probably mainly within the embryonic period [19].

The limitations of our study are the small numbers of subjects enrolled and the lack of comparison of the US findings with other imaging techniques such as microMRI applied to the same subject.
Further studies involving a larger sample size are needed to confirm the usefulness of 3D transvaginal US and HDlive rendering mode in future embryonic research and evaluation of fetal anomalies [10].

\section{Conclusions}

This study demonstrates that GE can be clearly visualized by 3D transvaginal US, and especially by HDlive rendering mode. The $3 \mathrm{D}$ transvaginal US has become the "golden standard" for in vivo morphological studies of the embryonic and early fetal brain. This method facilitated the development of a new field of research, neurosonoembryology. It also initiated new trends in embryology and developmental pathology, such as transferring the assessment of embryos and fetuses from in vitro studies to in vivo studies.

\section{Conflict of interest: none}

\section{References}

1. Tanaka H, Senoh D, Yanagihara T, Hata T. Intrauterine sonographic measurement of embryonic brain vesicle. Hum Reprod 2000; 15: 1407-1412.

2. Blaas HG, Eik-Nes SH. Sonoembryology and early prenatal diagnosis of neural anomalies. Prenat Diagn 2009; 29: $312-325$.

3. Kagan KO, Pintoffl K, Hoopmann M. First-trimester ultrasound images using HDlive. Ultrasound Obstet Gynecol 2011; 38: 607.

4. O'Rahilly R, Müler F. Significant features in the early prenatal development of the human brain. Ann Anat 2008; 190: 105-118.

5. Hansen DV, Lui JH, Flandin P, et al. Non-epithelial stem cells and cortical interneuron production in the human ganglionic eminences. Nat Neurosci 2013; 16: 1576-1587.

6. Müler F, O'Rahilly R. Embryonic Development of the Central Nervous System. In: Paxinos G, Jürgen KM (eds). The Human Nervous System, Second Edition. San Diego, California, USA Elsevier Academic Press 2004: 22-48.

7. Boitor-Borza D, Crivii C, Farcasanu S, Stamatian F. Morphology of the human brain in the embryonic period: anatomical study and assessment by $7 \mathrm{~T}$ magnetic resonance imaging. Obstetrica şi Ginecologia 2015; LXIII: 47-52.

8. Tanaka H, Hata T. Intrauterine sonographic measurement of the embryonic brain mantle. Ultrasound Obstet Gynecol 2009; 34: 47-51.

9. Gijtenbeek M, Bogers H, Groenenberg IA, et al. First trimester size charts of embryonic brain structures. Hum Reprod 2014; 29: 201-207.

10. Hata T, Hanaoka U, Tenkumo C, Sato M, Tanaka H, Ishimura M. Three- and four-dimensional HDlive render- 
ing images of normal and abnormal fetuses: pictorial essay. Arch Gynecol Obstet 2012; 286: 1431-1435.

11. Hull AD, James G, Salerno CC, Nelson T, Pretorius DH. Three-dimensional ultrasonography and assessment of the first-trimester fetus. J Ultrasound Med 2001; 20: 287-293.

12. Merz E, Abramovicz J, Baba K, et al. 3D imaging of the fetal face-recommendations from the International 3D Focus Group. Ultraschall Med 2012; 33: 175-182.

13. Hata T, Tenkumo C, Sato M, Kanenishi K, Ishimura M. Three-dimensional HDlive rendered images of intrauterine abnormalities during pregnancy. J Med Ultrasonics 2013; 40: 179-180.

14. Kurjak A, Kupesic S, Banovic I, Hafner T, Kos M. The study of morphology and circulation of early embryo by three-dimensional ultrasound and power Doppler. J Perinat Med 1999; 27: 145-157.
15. Feichtinger W. Transvaginal three-dimensional imaging. Ultrasound Obstet Gynecol 1993; 3: 375-378.

16. Zanforlin Filho SM, Araujo Junior E, Guiaraes Filho HA, Pires CR, Nardozza LM, Moron AF. Sonoembryology by three-dimensional ultrasonography: pictorial essay. Arch Gynecol Obstet 2007; 276: 197-200.

17. Pooh RK, Pooh K. Fetal neuroimaging by transvaginal 3D ultrasound and MRI. Donald School Journal of Ultrasound in Obstetrics and Gynecology 2011; 5: 23-32.

18. Pooh RK, Kurjak A. 3D/4D sonography moved prenatal diagnosis of fetal anomalies from the second to the first trimester of pregnancy. J Matern Fetal Neonatal Med 2012; 25: 433-455.

19. Shiota, K. Development and intrauterine fate of normal and abnormal human conceptuses. Congenit Anom 1991; 31: $67-80$. 